\title{
Evaluation of a Population-Based Program of Expedited Partner Therapy for Gonorrhea and Chlamydial Infection
}

\author{
MATTHEW R. GOLDEN, MD, MPH, ${ }^{*} \dagger$ JAMES P. HUGHES, PHD, †‡ DEVON D. BREWER, PHD§ \\ KING K. HOLMES, MD, PHD, † WILLIAM L. H. WHITTINGTON, AB, † MATTHEW HOGBEN, PHD,\| \\ CHERYL MALINSKI, BS, ${ }^{*}$ ANNE GOLDING, MPH, ${ }^{*}$ AND H. HUNTER HANDSFIELD, MD $†$
}

\begin{abstract}
Objective: To evaluate a partner notification program for gonorrhea and chlamydial infection that involves communitywide access to free patient-delivered partner therapy (PDPT) and use of case-report forms to triage patients to receive partner notification assistance.

Methods: We evaluated program components in randomly selected cases and compared outcomes before and after program institution.

Results: Following institution of the program, the percentage of cases who received PDPT from their diagnosing clinician increased from $5.6 \%$ to $16 \%$ (adjusted OR 3.2, 2.5-4.1). Among randomly selected cases, those referred to the health department via the casereport form were significantly more likely than nonreferred cases to have untreated sex partners (76\% vs. 35\%, OR 6.0, 95\% CI 4.5-8.0), to accept PDPT from the health department (36\% vs. 14\%, 3.3, 95\% CI 2.4-4.7), and to request that health department staff notify a partner for them (11\% vs. 3\%, OR 3.5, 95\% CI 1.8-6.7). The percentage of cases classified as having all of their partners treated increased from $39 \%$ to $65 \%$ concurrent with institution of the program.
\end{abstract}

Conclusions: A public health program that promotes routine use of PDPT and referral of selected patients for partner notification assistance appears to have improved partner notification outcomes.

PARTNER NOTIFICATION AND TREATMENT are mainstays of public health efforts to control bacterial sexually transmitted infections (STIs). In North America and parts of Europe, health department staff sometimes interview persons with STIs to facilitate their partners' treatment. However, existing resources are insufficient to provide public health partner notification services to all persons with gonorrhea or chlamydial infection in the United States, and fewer than $20 \%$ of cases reported in high morbidity areas are interviewed by public health workers. ${ }^{1}$

Dissatisfaction with the effectiveness of current approaches to partner notification coupled with the limited resources available to promote the activity prompted the US Institute of Medicine to advocate a fundamental redesign of partner notification services for STI. ${ }^{2}$ Expedited partner therapy (EPT), the practice of treating sex partners of persons with STIs without requiring the partners' prior clinical evaluation, is an alternative to traditional partner notification. In most

Dr. Golden was supported by NIH K23 AI01846. Research was funded by the Division of STD Prevention, National Center for HIV, STD, and TB Prevention, Centers for Disease Control and Prevention.

Correspondence: Matthew R. Golden, MD, MPH, Center for AIDS \& STD, Harborview Med Ctr, Box 359777, 325 9th Ave., Seattle, Washington 98109. E-mail: golden@u.washington.edu.

Received for publication August 3, 2006, and accepted November 8, 2006.
From the ${ }^{*}$ Public Health-Seattle and King County, Seattle, Washington; +Center for AIDS and STD, University of Washington, Seattle, Washington; the $\neq$ Department of Biostatistics, University of Washington, Seattle, Washington; \$Interdisciplinary Scientific Research, Seattle, Washington; and the UDivision of STD Prevention, Center for HIV, STD, and TB Prevention, Centers for Disease Control and Prevention, Atlanta, Georgia

instances, EPT includes patient-delivered partner therapy (PDPT), i.e., providing patients with medication to give to their sex partners. Approximately half of United States physicians sometimes use PDPT, but most do so infrequently. ${ }^{3-7}$ Three randomized controlled trials and 2 observational studies conducted in persons with gonorrhea or chlamydial infection have all found EPT to be equivalent or superior in assuring partner treatment to the more standard practice of asking patients to refer their partners for care..$^{8-13}$

Starting in 1998, our health department initiated a program using EPT as part of a randomized controlled trial. ${ }^{11,14}$ The results of that trial, our experience with EPT, and the need to improve the efficiency and scope of our public health partner notification efforts prompted us to develop a new, population-based partner notification program. In this paper, we describe and evaluate that program.

\section{Methods}

Beginning in 2004, Public Health-Seattle \& King County (PHSKC) initiated a new partner notification program for gonorrhea and chlamydial infection. This program involved 2 central components: (1) promotion of routine use of PDPT by clinicians treating heterosexuals with gonorrhea or chlamydial infection, including provision of free medication to all clinicians for use as PDPT; and (2) case report form-based triage of health department partner notification services. PHSKC does not encourage the routine use of PDPT among men who have sex with men (MSM) because of the relatively high prevalence of undiagnosed HIV among MSM seeking medical evaluation following exposure to gonorrhea or chlamydial infection, and because little data exist on the efficacy of PDPT in MSM. ${ }^{15}$

\section{STI Case Reports and Data Management}

The revised case report form asked reporting clinicians to check a box indicating one of 3 partner notification plans: (1) health department asked to assume responsibility of partner treatment; (2) clinician will ensure all partners are treated; or (3) all partners have 
already been treated. The form recommended that the clinician refer patients to the health department if any of the following factors were present: (1) patient had $>1$ sex partner in the preceding 60 days; (2) patient had a sex partner that (s)he does not plan to have sex with again; (3) the patient is unwilling or unable to contact a partner; or (4) the patient is a MSM. (We previously identified the first 2 factors as independent predictors of failure of persons with gonorrhea or chlamydial infection to notify partners. ${ }^{14}$ ) The form included instructions on how to obtain free PDPT.

A computer randomly selected cases for interview as they there entered into an electronic case registry and partner notification database. The proportion of cases selected varied over the course of the study period to maximize staff productivity without adding new staff. Overall, $70 \%$ of gonorrhea cases and $42 \%$ of chlamydial cases were randomly selected. (Gonorrhea cases were oversampled to assure adequate numbers for analysis.) Cases for which partner notification assistance was requested and all randomly selected cases were randomly assigned to one of 2 staff members for interview. Interviewers were blinded to whether providers requested partner notification assistance. PHSKC did not contact persons reported with STIs without the prior permission of the reporting clinician.

\section{Case Interviews and Provision of EPT by PHSKC Staff}

When contacted, index patients were interviewed about their sex partners during the 60 days preceding their STI testing. Persons reporting no partners during that period were questioned about their most recent partner. Index patients were offered PDPT for up to 3 partners and were offered assistance contacting partners they did not wish to notify themselves. With the exception of persons who reported drug allergies to standard therapies, staff offered all partners they contacted free medications available at commercial pharmacies. Staff advised partners to seek medical evaluation at the PSHKC STD Clinic or at another clinical venue. All packets included a single $1 \mathrm{~g}$ dose of azithromycin powder. Packets for the partners of persons with gonorrhea also contained either $400 \mathrm{mg}$ of cefixime or $400 \mathrm{mg}$ of cefpodoxime. We have previously described details related to the distribution of medications. ${ }^{11,14}$

\section{Evaluation and Statistical Methods}

We evaluated the performance of our new case-report form, use of PDPT, and the proportion of persons with untreated partners using the sample of randomly selected cases described above (Fig. 1). To assess trends in clinicians' use of PDPT, we compared the proportion of cases in the sample that received PDPT from their diagnosing clinician with the reported use of PDPT in a convenience sample of 5219 persons reported to PHSKC with gonorrhea or chlamydial infection between 1998 and 2003. The 1998-2003 sample was interviewed as part of a randomized controlled trial ${ }^{11,14}$ and reflects clinicians' use of PDPT before the trial intervention.

Following an initial interview during which cases were offered PDPT, we attempted to reinterview all persons who reported either of the 2 previously cited independent predictors of having untreated partners $(>1$ sex partner in the 60 days before diagnosis, or a sex partner with whom they did not intend to have sex in the future). We regarded a partner as treated if (s)he met any of the following criteria: (1) index case reported that the partner was treated; (2) PHSKC prescribed PDPT for the partner and the index patient received a partner packet and reported giving it to the partner; (3) staff directly spoke to the partner who stated (s)he had been treated or had received a partner packet; or (4) PHSKC

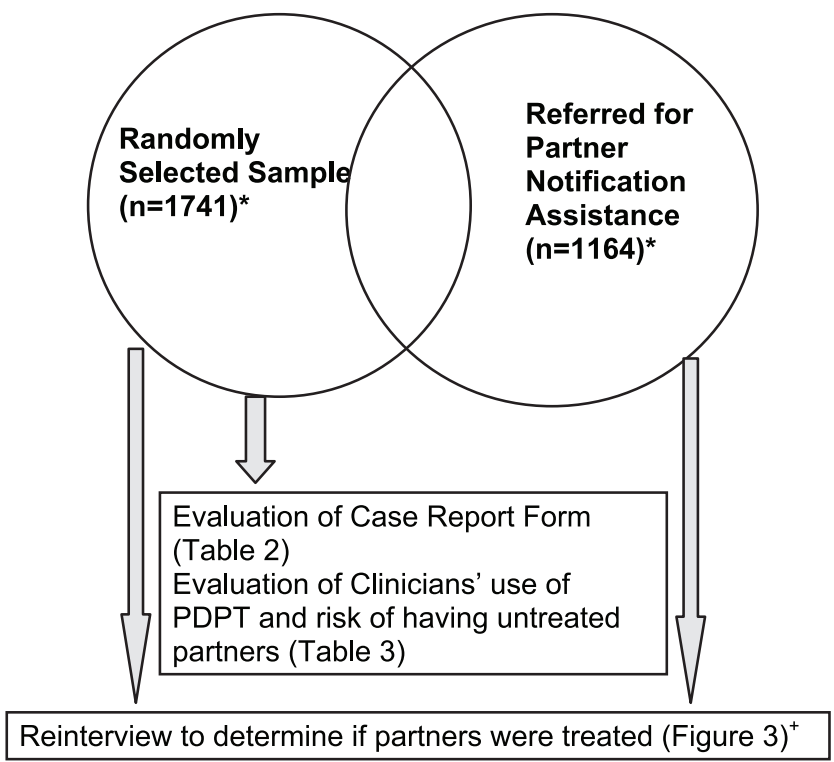

Fig. 1. Organization of study analysis.

prescribed PDPT for the partner, the index patient retrieved a partner packet from a pharmacy, and the index patient did not meet our criteria for reinterview. In a previous study, ${ }^{11}$ we found that $224(96 \%)$ of 234 persons meeting these criteria reported that they were certain their partner was treated [unpublished data]. We classified partner(s) who did meet these criteria as untreated.

We performed bivariate tests of association using $\chi^{2}$ and logistic regression and multivariate analysis using logistic regression. Because we oversampled cases of gonorrhea and because the sample of interviewed persons differed from the randomly selected sample, we weighted estimates of the proportion of partners receiving PDPT and the proportion with untreated partners at time of initial partner notification interview. Specifically, we used logistic regression to predict the probability that a case in the randomly selected sample was interviewed as a function of age, gender, STI, type of diagnosing facility, and mechanism of case reporting and case report partner notification plan. The weights were then set proportional to the inverse of these predicted probabilities. We also used these weights in analyses evaluating factors associated with providers' use of PDPT and with having untreated partners (Table 2).

To estimate the population-level impact of the program, we first estimated the proportion of cases with no untreated partners under a program of PDPT but without additional public health intervention. This estimate was defined using the observed proportion in persons interviewed $>7$ days after their treatment in the 20042005 random interview sample, weighted for nonresponse and oversampling of gonorrhea cases. This is the middle bar in Figure 2. We then estimated the impact of improved partner notification by providers, including increased provider use of PDPT, on the proportion of persons with untreated partners by comparing the 1998-2003 and the 2004-2005 samples. To do this, we combined the 2 datasets and fit a logistic regression with "any untreated partner" as the outcome and gender, age, STI, type of diagnosing facility, and sample time period as covariates for persons interviewed $>7$ days after their treatment. We combined the (adjusted) odds ratio for cohort time period from this regression with the middle bar in Figure 2 to produce the first bar in Figure 2. Finally, we estimated the additional effect of the public health intervention as (1) the difference between the proportion of partners treated at 
TABLE 1. Demographic Characteristics, STD Diagnoses, Source of STD Diagnosis, and Partner Notification Plan Among a Random Sample of Nonincarcerated Heterosexuals Reported to PHSKC STD Clinic, May 2002-August 2005

\begin{tabular}{|c|c|c|}
\hline Characteristic & $\begin{array}{c}\text { Random } \\
\text { Sample } \\
(\mathrm{n}=3,747) \\
\mathrm{N}(\%)\end{array}$ & $\begin{array}{c}\text { Interviewed } \\
\text { Cases } \\
(n=1,757) \\
N(\%)\end{array}$ \\
\hline \multicolumn{3}{|l|}{ Sex } \\
\hline Female & $2,699(72)$ & $1,353(77)$ \\
\hline Male & $1,048(28)$ & 404 (23) \\
\hline \multicolumn{3}{|l|}{ Patient age } \\
\hline$\leq 19$ & $1,263(34)$ & $598(34)$ \\
\hline $20-24$ & $1,218(33)$ & $611(35)$ \\
\hline $25-29$ & $591(16)$ & $279(16)$ \\
\hline$>29$ & $663(18)$ & 264 (15) \\
\hline \multicolumn{3}{|l|}{ STI } \\
\hline Gonorrhea & $601(16)$ & $236(13)$ \\
\hline Chlamydia & 2,908 (78) & $1,412(80)$ \\
\hline Both & $238(6)$ & $109(6)$ \\
\hline \multicolumn{3}{|l|}{ Clinical setting N (\%) } \\
\hline Public health & 479 (13) & $135(8)$ \\
\hline Family planning & $548(15)$ & $236(13)$ \\
\hline STD clinic & $372(10)$ & 209 (12) \\
\hline Emergency room & $266(7)$ & $138(8)$ \\
\hline Private sector & $2082(56)$ & 1,039 (59) \\
\hline \multicolumn{3}{|l|}{$\begin{array}{l}\text { Case-reporting mechanism and } \\
\text { PN plan indicated on } \\
\text { case-report form }\end{array}$} \\
\hline New case report form used & $2,358(63)$ & $1,049(60)$ \\
\hline All partners already treated & $454(19)$ & $134(13)$ \\
\hline Provider to assure PN & $615(26)$ & 195 (19) \\
\hline $\begin{array}{l}\text { Health department to } \\
\text { assure PN }\end{array}$ & $1,037(44)$ & $655(62)$ \\
\hline Missing & $252(11)$ & $65(6)$ \\
\hline Old case report form used ${ }^{*}$ & $527(14)$ & $225(13)$ \\
\hline $\begin{array}{l}\text { No case report form } \\
\text { submitted (laboratory } \\
\text { report only) }\end{array}$ & 490 (13) & $274(16)$ \\
\hline STD clinic case ${ }^{*}$ & $372(10)$ & $209(12)$ \\
\hline
\end{tabular}

*No provider PN plan available. PN plans were not recorded on case-report forms distributed by PHSKC before April 2004, and only electronic case reports were used in the PHSKC STD clinic. Between May 2004 and August 2005, the percentage of all cases reported on old case-report forms decreased from $30 \%$ to $13 \%$.

$>7$ days after treatment and the final proportion of partners treated among cases who were interviewed; and (2) zero in cases who were not interviewed. Statistical analyses were performed using the SAS system and Stata version 9.

\section{Results}

\section{Study Population}

Between May 2004 and August 2005, King County clinicians reported 10,791 cases of gonorrhea or genital chlamydial infection. Analysis of the new partner notification system excluded 2715 cases $(25 \%)$ for the following reasons: incarceration $(n=724)$, men who have sex with men $(n=1229)$, residency outside of King County $(n=348)$, inability to speak English $(n=248)$, case reported $>30$ days after treatment $(\mathrm{n}=52)$, sexual assault $(\mathrm{n}=$ $28)$, age $<14(\mathrm{n}=16)$, and other reasons $(\mathrm{n}=70)$.

Of the remaining 8076 cases, 3747 (46\%) were randomly selected for interview. Reporting clinicians granted PHSKC permission to contact $2812(75 \%)$ of these cases, of whom 2165 (58\% of

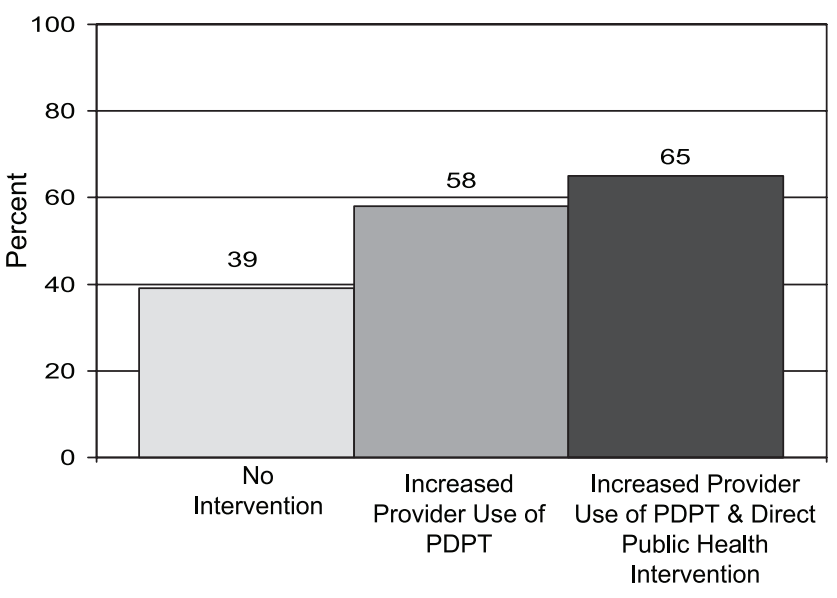

Fig. 2. Estimated percentage of cases of gonorrhea or chlamydial infection for whom all partners were treated in the absence of a public health partner notification program, with a program involving only use of new case report forms and promotion of PDPT use by diagnosing providers, and with a program involving new case report forms, promotion of PDPT use by providers and direct public health outreach.

total) were contacted, and 1757 (47\% of total) agreed to be interviewed. Table 1 summarizes the characteristics of interviewed and not interviewed cases. In multivariate analysis comparing interviewed persons to those who were not interviewed, interviewees were significantly younger, more often had chlamydial infection alone, and were more often female. The 2 groups also differed significantly in the distribution of the partner notification plans indicated by providers and case-reporting mechanism.

\section{Case Report Form Categorization of Partner Notification Plans}

Clinicians used the new case report form to report 1049 interviewed persons who were part of the random sample (Table 2). On bivariate analysis comparing index cases for whom clinicians requested partner notification assistance to those for whom it was not requested (excluding persons for whom no partner notification plan was indicated), cases triaged to receive health department assistance were significantly more likely to have multiple partners or partners with whom they did not plan to have sex again (OR 2.4, 95\% CI 1.8-3.1). These persons were also significantly more likely to have untreated partners (OR 6.0, 95\% CI 4.5-8.0), to accept PDPT from the health department (OR 3.3, 95\% CI 2.4$4.7)$, and to request that health department staff notify a partner for them (OR $3.595 \%$ CI 1.8-6.7); they were significantly less likely to have received PDPT from their diagnosing provider before their partner notification interview (OR 0.11, 95\% CI 0.07-0.16).

\section{Providers' Use of PDPT and the Percentage of Persons With Untreated Sex Partners Before Interview}

Overall, $16 \%$ of all patients received PDPT from their diagnosing clinician (Table 3). In the multivariate model, provision of PDPT by diagnosing clinicians was more common for female index patients, in family planning clinics and the STD clinic, and when providers indicated on the case report form that all partners had already been treated or that the provider planned to arrange partner notification themselves. Providers used PDPT significantly less often with patients diagnosed in emergency rooms and with patients who met criteria for referral to the health department for partner notification assistance. 
TABLE 2. Presence of Risk Criteria for Referral for Health Department Partner Notification and Partner Notification Outcomes Among Randomly Selected Cases of Gonorrhea or Chlamydial Infection by How Reporting Providers Indicated They Planned to Manage Partners on Case Report Form

PN Plan Indicated on Case Report Form

\begin{tabular}{|c|c|c|c|c|}
\hline & \\
\hline & $\begin{array}{l}\text { All Partner Already Treated } \\
\qquad(\mathrm{n}=134) \mathrm{N}(\%)\end{array}$ & $\begin{array}{l}\text { Provider to Assure PN } \\
\quad(n=195) N(\%)\end{array}$ & $\begin{array}{l}\text { Health Department } \\
\text { Asked to Assure PN } \\
(n=655) N(\%)\end{array}$ & $\begin{array}{l}\text { PN Plan Left Blank } \\
(\mathrm{n}=65) \mathrm{N}(\%)\end{array}$ \\
\hline$>1$ Sex partner & $26(20)$ & $47(24)$ & $213(33)$ & $18(28)$ \\
\hline $\begin{array}{l}\text { Patient does not plan to have sex } \\
\text { with } \geq 1 \text { of his/her partners } \\
\text { again }\end{array}$ & $39(32)$ & $72(41)$ & $347(59)$ & $28(47)$ \\
\hline $\begin{array}{l}\text { Index patient received PDPT from } \\
\text { provider prior to public health } \\
\text { interview by public health staff }\end{array}$ & $39(28)$ & $79(40)$ & $37(6)$ & $5(8)$ \\
\hline $\begin{array}{l}\text { Any untreated partner at time of } \\
\text { public health interview }\end{array}$ & $37(28)$ & $79(40)$ & $501(76)$ & $38(58)$ \\
\hline Accepted PDPT from health dept. & $15(11)$ & $28(15)$ & $227(36)$ & $18(28)$ \\
\hline
\end{tabular}

The percentage of cases with untreated sex partners decreased with longer intervals between the date of index patient treatment and the date of their initial partner notification interview; at intervals of 0,1 to 4,5 to 7,8 to 13 , and $\geq 14$ days, $88 \%, 71 \%, 51 \%$, $45 \%$, and $47 \%$ reported having at least 1 untreated partner, respectively. In multivariate analysis restricted to persons interviewed $>7$ days after treatment, reporting an untreated partner was significantly associated with patient age $<20$ years, having one of the 2 risk factors for partner notification failure indicated on the case-report form, and with not receiving PDPT from a diagnosing provider (Table 3). Cases were less likely to report having untreated partners if the case report form indicated either that they had no untreated partners or that the diagnosing provider would manage partner notification themselves.

Trends in the Use of PDPT and Number of Persons With Untreated Partners

To assess trends in the use of PDPT and the effect of PDPT on the proportion of persons with untreated sex partners, we compared data from 2004 to 2005 with similar data collected from 1998 to 2003, before we instituted our new partner management system. Among persons interviewed $>7$ days after treatment, controlling for index patient gender, STI diagnosis, type of diagnosing clinical venue, and the presence of case report risks for partner notification failure, cases reported in 2004-2005 were more likely to have received PDPT from a provider (5.6\% vs. $16 \%$, OR 3.2, 2.5-4.1). In a separate multivariate model controlling for the same factors, persons in the 2004-2005 group were less likely to report having untreated partners than were persons in 1998 2003 group (OR 0.63 , 95\% CI $0.52-0.74$ ). The association between diagnosis in 2004-2005 and having untreated partners continued to be significant when PDPT use by providers was included in the model (OR $0.76,95 \%$ CI $0.63-0.91$ ).

\section{Partner Management by Public Health}

PHSKC staff contacted and interviewed 2396 (30\%) of the 8076 nonincarcerated heterosexuals without specific exclusion criteria who had been diagnosed with gonorrhea or chlamydial infection during the evaluation period. A total of 1529 cases (64\%) reported having $\geq 1$ untreated sex partner, of whom 641 (42\%) accepted PDPT to treat 695 (28\%) of the 2458 untreated sex partners. Of 2458 partners classified as being untreated at time of initial interview, $1263(51 \%)$ were subsequently treated, including $498(72 \%)$ of the initially untreated 695 partners for whom index cases accepted PDPT. (Index patients retrieved partner packets for an additional 177 partners for whom final treatment status was unknown because of unsuccessful reinterview efforts; these persons were classified as being untreated.) A total of 167 index patients with untreated partners $(11 \%)$ requested that public health staff notify 200 sex partners, of whom $136(68 \%)$ were treated. Combining all these outcomes, we classified as treated $2461(66 \%)$ of the total 3710 partners acknowledged by interviewed patients; partner notification efforts led to successful treatment of all potentially exposed partners of 1837 (77\%) interviewed persons.

\section{Estimated Impact of Increased PDPT and Public Health Outreach}

Figure 2 shows the estimated increase in the proportion of cases all of whose partners were treated as a result of the partner notification program. In the absence of any public health partner notification program, we estimate that $39 \%$ of cases would have had all of their partners treated. Improved partner notification by diagnosing providers, including increased use of PDPT, increased the percentage of cases whose partners were all treated to $58 \%$. Direct intervention by public health partner notification staff increased this proportion to $65 \%$.

\section{Discussion}

Building on a series of randomized trials demonstrating that EPT can improve partner notification outcomes ${ }^{10-12}$ as well as a study defining risk factors for partner notification failure, ${ }^{14}$ we developed a population-based public health program for partner 
TABLE 3. Factors Associated With Diagnosing Clinician's Use of PDPT and With Having At least 1 Untreated Sex Partner $>7 \mathrm{~d}$ After Index Patient Treatment in 1,757 Randomly Selected Heterosexuals Reported to PHSKC, May 2004-August 2005*

\begin{tabular}{|c|c|c|c|c|c|c|}
\hline \multirow[b]{2}{*}{ Characteristic } & \multicolumn{3}{|c|}{ Receipt of PDPT from Diagnosing Clinician } & \multicolumn{3}{|c|}{$\begin{array}{l}\text { Any Untreated Sex Partner Among Persons } \\
\text { Interviewed }>7 \text { d After Treatment }\end{array}$} \\
\hline & Percent & $\begin{array}{l}\text { Bivariate OR } \\
(95 \% \mathrm{Cl})\end{array}$ & $\begin{array}{l}\text { Multivariate OR } \\
(95 \% \mathrm{Cl})\end{array}$ & Percent & $\begin{array}{l}\text { Bivariate OR } \\
(95 \% \mathrm{Cl})\end{array}$ & $\begin{array}{l}\text { Multivariate OR } \\
\quad(95 \% \mathrm{Cl})\end{array}$ \\
\hline All persons & 16 & NA & NA & 42 & NA & NA \\
\hline \multicolumn{7}{|l|}{ Sex } \\
\hline$\leq 19$ & 19 & $2.2(1.4-3.3)$ & $1.6(0.96-2.7)$ & 47 & $1.2(0.80-1.8)$ & $2.1(1.2-3.7)$ \\
\hline $20-24$ & 18 & $2.0(1.3-3.0)$ & $1.3(0.77-2.1)$ & 41 & $0.94(0.62-1.4)$ & $1.4(0.81-2.3)$ \\
\hline $25-29$ & 14 & $1.5(0.89-2.4)$ & $0.99(0.56-1.7)$ & 38 & $0.82(0.52-1.3)$ & $1.4(0.77-2.4)$ \\
\hline$>29$ & 10 & 1.0 & 1.0 & 42 & 1.0 & 1.0 \\
\hline \multicolumn{7}{|l|}{ STI } \\
\hline Gonorrhea & 14 & $0.80(0.55-1.2)$ & $0.90(0.58-1.4)$ & 51 & $1.5(1.0-2.2)$ & $1.0(0.65-1.7)$ \\
\hline Emergency room & 0.6 & $0.043(0.004-0.44)$ & $0.09(0.01-0.96)$ & 64 & $2.4(1.3-4.3)$ & $1.5(0.73-3.0)$ \\
\hline Private sector & 12 & 1.0 & 1.0 & 43 & 1.0 & 1.0 \\
\hline \multicolumn{7}{|l|}{ PN plan on case report form } \\
\hline \multicolumn{7}{|l|}{ New case report form used } \\
\hline All partners already treated & 25 & $3.8(2.1-6.8)$ & $2.1(1.2-3.9)$ & 22 & $0.38(0.21-0.65)$ & $0.35(0.17-0.72)$ \\
\hline Provider to assure PN & 41 & $8.0(4.8-13.5)$ & $4.1(2.4-7.2)$ & 25 & $0.43(0.26-0.73)$ & $0.39(0.19-0.82)$ \\
\hline Health dept. to assure PN & 6 & $0.67(0.36-1.2)$ & $0.49(0.27-0.9)$ & 59 & $1.8(1.2-2.9)$ & $0.80(0.44-1.4)$ \\
\hline Missing & 6 & $0.76(0.32-1.8)$ & $0.70(0.28-1.8)$ & 48 & $1.2(0.66-2.1)$ & $0.90(0.43-1.9)$ \\
\hline No case report form submitted & 9 & $1.2(0.6-2.2)$ & $0.68(0.36-1.3)$ & 53 & $1.5(0.88-2.5)$ & $0.62(0.34-1.1)$ \\
\hline STD clinic case & 25 & $3.8(2.1-6.8)$ & $\mathrm{NA}^{+}$ & 42 & $0.94(0.49-1.8)$ & $\mathrm{NA}^{\dagger}$ \\
\hline Old case report form used & 9 & 1.0 & 1.0 & 44 & 1.0 & 1.0 \\
\hline \multicolumn{7}{|l|}{ Risk factor on case report } \\
\hline Present & 12 & $0.45(0.34-0.58)$ & $0.52(0.39-0.71)$ & 63 & $6.5(4.6-9.0)$ & $7.2(4.9-10.5)$ \\
\hline
\end{tabular}

*Analyses are weighted to reduce response bias as described in the methods section. Data are weighted for age, gender, STI diagnosis, and partner notification plan and reporting mechanism.

${ }^{\top}$ STD clinic as diagnosing site is included in the model under type of diagnosing clinic.

management of persons with gonorrhea or chlamydial infection that promotes widespread use of publicly financed PDPT and utilizes provider-initiated case report forms to triage patients at high risk for partner notification failure to receive public health partner notification assistance. Evaluating the effectiveness of this program, we found that: (1) the use of PDPT by providers in King County increased almost 3 -fold concurrent with implementation of the program; (2) patients who received PDPT from their provider were significantly less likely than other recently infected persons to report having untreated partners; (3) providers successfully used the modified case report form to selectively refer patients with untreated partners to public health; (4) patients referred to public health by their providers usually accepted some form of partner management assistance; and (5) the estimated percentage of persons with gonorrhea or chlamydial infection for whom all partners were treated rose from $39 \%$ to $65 \%$ concurrent with the institution of the partner notification program.

Public health officials interested in replicating our approach should be aware that it requires groundwork and infrastructure. First, before initiating our program, we worked with our State Boards of Pharmacy and of Medical Quality Assurance to establish that PDPT was legal in Washington State. ${ }^{15}$ The legal status of PDPT is uncertain in much of the United States. ${ }^{16}$ Second, our approach to assigning cases and tracking partner notification plans required us to have a real-time electronic case registry, and to enhance laboratory-based reporting to ensure that we knew about cases near the time of diagnosis. Although many States maintain electronic case registries and receive laboratory reports, such systems are not universal, and electronic case registry databases are often disconnected from partner notification activities. Third, treating large numbers of partners requires a mechanism for distributing medications. To do this, we arranged for local pharmacy chains to distribute partner packets for public health purposes. While we are not aware of other health departments with similar arrangements, we believe this model can be replicated. Moreover, once established, this collaboration could potentially be used for other public health activities, such as vaccination and mass distribution of medications in an emergency. Finally, although some areas might adopt much of our approach by reassigning existing public health personnel to perform new functions, most areas would likely require either new funding or diversion of existing funds from other activities. Cost-effectiveness analyses suggest that EPT is probably more cost-effective than other approaches to 
gonorrhea and chlamydial case finding, ${ }^{17}$ though additional research in this area is needed.

Our study has several limitations. We cannot be certain that the increased use of PDPT and the other benefits that we observed were direct consequences of our intervention. However, we observed no change in the use of PDPT among providers in King County between 1998 and 2004,3,11 suggesting that a trend toward increased use was not ongoing before institution of our program. Also, our findings regarding the proportion of cases receiving PDPT from providers and the proportion of cases with untreated partners represent estimates. The precision of these estimates is limited by the fact that we were not able to interview everyone in our random sample, and by our inability to definitively ascertain the treatment status of all partners. We attempted to limit inaccuracies in our estimates by weighting our analyses of interviewed patients to reflect the composition of the entire random sample. However, we cannot completely eliminate the possibility that some of our findings may reflect bias for which we have not adjusted.

In conclusion, in a population-based study, we found that a public health partner notification program that promotes providers' use of publicly financed PDPT and employs case-report forms to triage selected patients to receive partner notification assistance can increase the proportion of cases of gonorrhea or chlamydial infection whose partners are treated. The cost-effectiveness of this system, its impact on the prevalence of infection in the population, approaches to further increase providers' use of PDPT, and the feasibility of widely instituting similar programs in other areas remain unknown. We are currently studying these issues in a community-level randomized trial in Washington State. However, given the failure of current partner notification efforts to assure the treatment of many patients' sex partners and the persistence of unacceptably high levels of STI morbidity in the United States and many other high-income nations, we believe our approach merits additional evaluation in other settings.

\section{References}

1. Golden MR, Hogben M, Handsfield HH, et al. Partner notification for HIV and STD in the United States: Low coverage for gonorrhea, chlamydial infection, and HIV. Sex Transm Dis 2003; 30:490-496.

2. Committee on Prevention and Control of Sex Transmission Disease, Institute of Medicine (U.S.). In: Eng TR, Butler WT, eds. The Hidden Epidemic: Confronting Sexually Transmitted Diseases. Washington, DC: National Academy Press; 1997.

3. Golden MR, Whittington WL, Gorbach PM, et al. Partner notification for chlamydial infections among private sector clinicians in SeattleKing County: A clinician and patient survey. Sex Transm Dis 1999; 26:543-547.
4. St Lawrence JS, Montano DE, Kasprzyk D, et al. STD screening, testing, case reporting, and clinical and partner notification practices: A national survey of US physicians. Am J Public Health 2002; 92:1784-1788.

5. Hogben M, McCree DH, Golden MR. Patient-delivered partner therapy for sex transm dis as practiced by U.S. physicians. Sex Transm Dis 2005; 32:101-105.

6. Niccolai LM, Winston DM. Physicians' opinions on partner management for nonviral sexually transmitted infections. Am J Prev Med $2005 ; 28: 229-233$.

7. Rogers ME, Opdyke KM, Blank S, et al. Patient-delivered partner treatment and other partner management strategies for sexually transmitted diseases used by New York city healthcare providers. Sex Transm Dis, in press.

8. Ramstedt K, Forssman L, Johannisson G. Contact tracing in the control of genital Chlamydia trachomatis infection. Int J STD AIDS 1991; 2:116-118.

9. Kissinger P, Brown R, Reed K, et al. Effectiveness of patient delivered partner medication for preventing recurrent Chlamydia trachomatis. Sex Transm Infect 1998; 74:331-333.

10. Schillinger JA, Kissinger P, Calvet H, et al. Patient-delivered partner treatment with azithromycin to prevent repeated Chlamydia trachomatis infection among women: A randomized, controlled trial. Sex Transm Dis 2003; 30:49-56.

11. Golden MR, Whittington WL, Handsfield HH, et al. Effect of expedited treatment of sex partners on recurrent or persistent gonorrhea or chlamydial infection. N Engl J Med 2005; 352:676-685.

12. Kissinger P, Mohammed H, Richardson-Alston G, et al. Patientdelivered partner treatment for male urethritis: A randomized, controlled trial. Clin Infect Dis 2005; 41:623-629.

13. CDC. Expedited Partner Therapy in the Management of Sexually Transmitted Diseases. Atlanta, GA: US Department of Health and Human Services; 2006.

14. Golden MR, Whittington WL, Handsfield HH, et al. Partner management for gonococcal and chlamydial infection: Expansion of public health services to the private sector and expedited sex partner treatment through a partnership with commercial pharmacies. Sex Transm Dis 2001; 28:658-665.

15. Stekler J, Bachmann L, Brotman RM, et al. Concurrent sexually transmitted infections (STIs) in sex partners of patients with selected STIs: Implications for patient-delivered partner therapy. Clin Infect Dis 2005 40:787-793. CDC website: http://www.cdc.gov/std/ept/legal/default.htm.

16. Golden MR, Anukam U, Williams DH, et al. The legal status of patient delivered partner therapy for sexually transmitted infections in the United States: A national survey of state medical and pharmacy boards. Sex Transm Dis 2005; 32:112-114.

17. Gift TL, Farley TA, Leichliter JS, et al. The cost-effectiveness of patient delivered partner treatment (PDPT) for female partners of men with urethritis compared to two alternatives: New Orleans 2001-2004. Paper presented at 16th Biennial Meeting of the International Society for Sexually Transmitted Disease Research, Amsterdam, Netherlands, 2005. 